\title{
Dynamics of Commitment and Contribution Quality in Collaborative Communities
}

\author{
Markus Schaal \\ Collaborative Systems Group \\ Bilkent University, Ankara, Turkey \\ Email:schaal@cs.bilkent.edu.tr
}

\author{
Yavuz Eren \\ Research and Development \\ GeoTech Group, Ankara, Turkey \\ Email: yeren@ags-group.com
}

\begin{abstract}
It is well-known that commitment is an important ingredient for contributions of high quality. With the internet heading towards being an actionable social space, rather than a collection of web-sites, the issue of quality becomes crucial for sharing knowledge and action in collaborative scenarios.

We propose a case study for the investigation of the influence of time, user feedback and interface complexity on contribution quality. We plan to exploit different online user groups across two different temporal phases for the evaluation.
\end{abstract}

\section{INTRODUCTION}

\section{A. Background}

The Internet is going to become an actionable social space centered around people, places, artefacts and actions. This is reflected by recent developments like Semantic Web [3], Web 2.0 or the emergence of a growing number of collaborative web sites [14], where users can share photos, videos, stories, or edit an encyclopedia in a collaborative manner.

Therefore, we expect the nature of the internet to change dramatically. Instead of webservers, that provide content to a number of clients, we expect the clients to create and share their identities and information space with others in a seamless manner, not bound to particular web-sites.

The notion of commitment and information quality will become crucial for this internet of the future. Anonymity on the web - despite its advantages - has led to Wikipedia abuses, faked identities and false content in so-called Web 2.0 applications. In order to overcome these problems, communication protocols must consider the level of trust and commitment while opening channels for seamless sharing of concepts, ideas and personal information.

Our long-term aim is the creation of protocols that prevent non-collaborative/ non-committed users from entering their wrong or inappropriate content into the space of shared concepts while the collaborative/ committed user can easily enter such content, share it with others while increasing his trust value and thus his benefits from the collaboration in the most optimal way.

\section{B. The Goal}

The goal of the proposed research is to study the change in quality of user input to a collaborative web-site over the course of time and with regard to changes in the interface complexity or awareness of feedback mechanisms.
In a nutshell the proposed case study is a web portal which allows for its members to post images and descriptions of problematic urban structures (traffic lights, potholes etc.), prompting other users into adding comments and solutions in a forum format.

We will observe user behavior and quality of contribution through time while increasing the complexity of the user interaction space for certain test groups - and having control groups without such improvements. We distinguish two temporal phases: Phase I prior to some change for certain users and Phase II thereafter. We distinguish the following user groups:

1) Anonymous Users: They can look up the problems and solutions, but they must register for contributions. There is no change for anonymous users between Phases I and II.

2) General Users: A general user will have a simple user interface and will not be able to give feedback. Some general users (of Phase I) will become power users in Phase II.

3) Feedback Users: Same as general user, but with the ability to give feedback about other users' contributions. Some feedback users (of Phase I) will become power feedback users in Phase II. Feedback users will also benefit from the results of feedback: Their contents will be sorted accordingly.

4) Power Users: A power user will use a complex user interface and will not be able to give feedback.

5) Feedback Power Users: Same as power user, but with the ability to give feedback about other users' contributions, cf. Feedback Users.

During the first phase of the project all registered users use the same simple interface for data entry. Due to a random selection, one half of the users will be feedback users, the others are general users. The switch to the second phase is realized by upgrading half of the users to become Power Users, i.e. half of the Feedback Users become Feedback Power Users and half of the General Users become Power Users (but without feedback facility). The change in contribution quality will be studied across time and across groups within the same temporal phase. This is depicted in Fig. 1. 


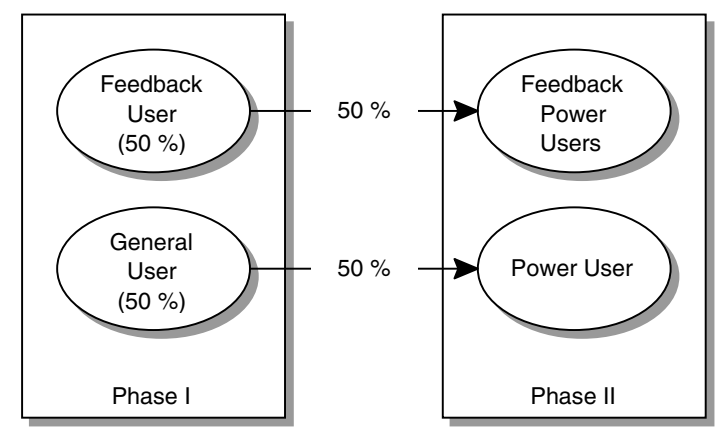

Fig. 1. Case Study Overview

We conjecture, that high interface complexity ${ }^{1}$ is correlated with high commitment and high contribution quality. This can be tested directly from the expected data. For the more sophisticated conjecture that users may even increase their commitment if interface complexity is increased, we will also need a control group for comparing losses during the second phase.

The following conjectures are to be tested:

- Conjecture 1: Quality of contributed content correlates positively with interface complexity.

- Conjecture 2: Increase of interface complexity correlates positively with increased quality of contributed content.

- Conjecture 3: Time correlates positively with quality of contributed content, i.e. quality increases over time.

- Conjecture 4: Quality of contributed content correlates positively with awareness of feedback.

For the dynamic assessment of contributed quality, we will exploit user feedback and a computational model based on Bayesian Belief Update, see Section III.

In order to conduct the study and the analysis, an interactive web-site will be created, e.g. by using an RIA (Rich Internet Application) approach made possible by technologies such as Flex 2 from Adobe $^{2}$.

\section{RELATED WORK}

Web Portals with collaboratively working online communities have already attracted researchers from many fields. Leuf and Cunningham [7] introduced the Wiki Way as a means for collaborative editing of hypertext. Viégas et al. [15] introduce so-called History Flow Visualizations for the analysis of cooperation and conflict in Wikipedia. Giles [4] compares Wikipedia with other well-known encyclopedias. Holloway et al. [5] visualize the semantic coverage of Wikipedia.

Recently, the power of knowledge explication became apparent and about to be termed as human computing, cf. e.g. [16] or [14]. First systematic studies of user behavior are conducted, e.g. [8].

The importance of trust for the sematic web has been recognized [11] and many models for the propagation and

\footnotetext{
${ }^{1}$ We are not interested in unneeded complexity, but in complexity that comes with increased functionality.

${ }^{2}$ cf. http: / /www. adobe.com/products/flex/
}

update of trust have been proposed, e.g. [13]. Similar and more mature approaches can be found for Multi-Agent-Systems (MAS), cf. [12] [2].

The relationship of commitment and online community contribution has been studied in [6] and [1], but not with a focus on interface complexity or feedback.

Incentives for knowledge sharing have been studied in Müller [9]. Even though not directly related to quality of contributions, they provide a rich model for the dependencies between cultural factors and incentives and their influence on commitment and contribution.

\section{THE USER MODEL}

The user's qualities are to be evaluated over time. Opening of a new problem thread, commenting on an existing thread, proper feedback and consistence are different aspects of the same user and should be modeled separately. We employ the following random variables for these qualities and apply Bayesian Belief Updates for the update of those qualities based on actual user feedback.

- Relevance $R_{u}$ per user, a binary variable with values high and low. The probability $P\left(R_{u}=h i g h\right)$ corresponds to the likelihood that the user will open a relevant thread.

- Quality $Q_{u}$ per user, a binary variable with values strong and weak. The probability $P\left(Q_{u}=\right.$ strong $)$ corresponds to the likelihood that a user will post an item of high perceived quality

- Consistency $C_{u}$ per user, a binary variable with values high and low. The probability $P\left(C_{u}=\right.$ high $)$ corresponds to the likelihood that the user will create postings with relevance and quality as assigned to the user

- Honesty $H_{u}$ per user, a binary variable with values honest and dishonest. The probability $P\left(H_{u}=\right.$ honest $)$ corresponds to the likelihood that the user will give honest feedback

For the update of those variables, we rely on a Bayesian Belief Network (BBN) and collect evidence after a certain time interval for Bayesian Belief Updates, see [13].

After the opening of a problem, relevance-votings will be accepted for a certain time interval. After the posting of a refinement/ argument or solution, quality-votings will be accepted for a certain time interval. Subsequently, the model of the users will be updated accordingly.

The Bayesian Belief Network for the single users quality (node Author), consistency (node Cons.) and Posting quality, together with the honesty of other users in a vote (nodes Voter $x$ ) and their votes (nodes Vote $x$ ) is shown in Fig. 2, cf. [10]. Initially, all probabilities are 0.5 . The posting quality is conditioned on the authors quality and consistency, with full dependence $(1.0 / 0.0)$ in the case of high consistency and ignorance $(0.5 / 0.5)$ in the case of low consistency. A similar table applies for the conditioning of a single vote on the posting quality and the honesty of the voter, only the full dependence in the case of a honest voter has been weakened to $(0.9 / 0.1)$ in order to credit for non-deliberate error. 


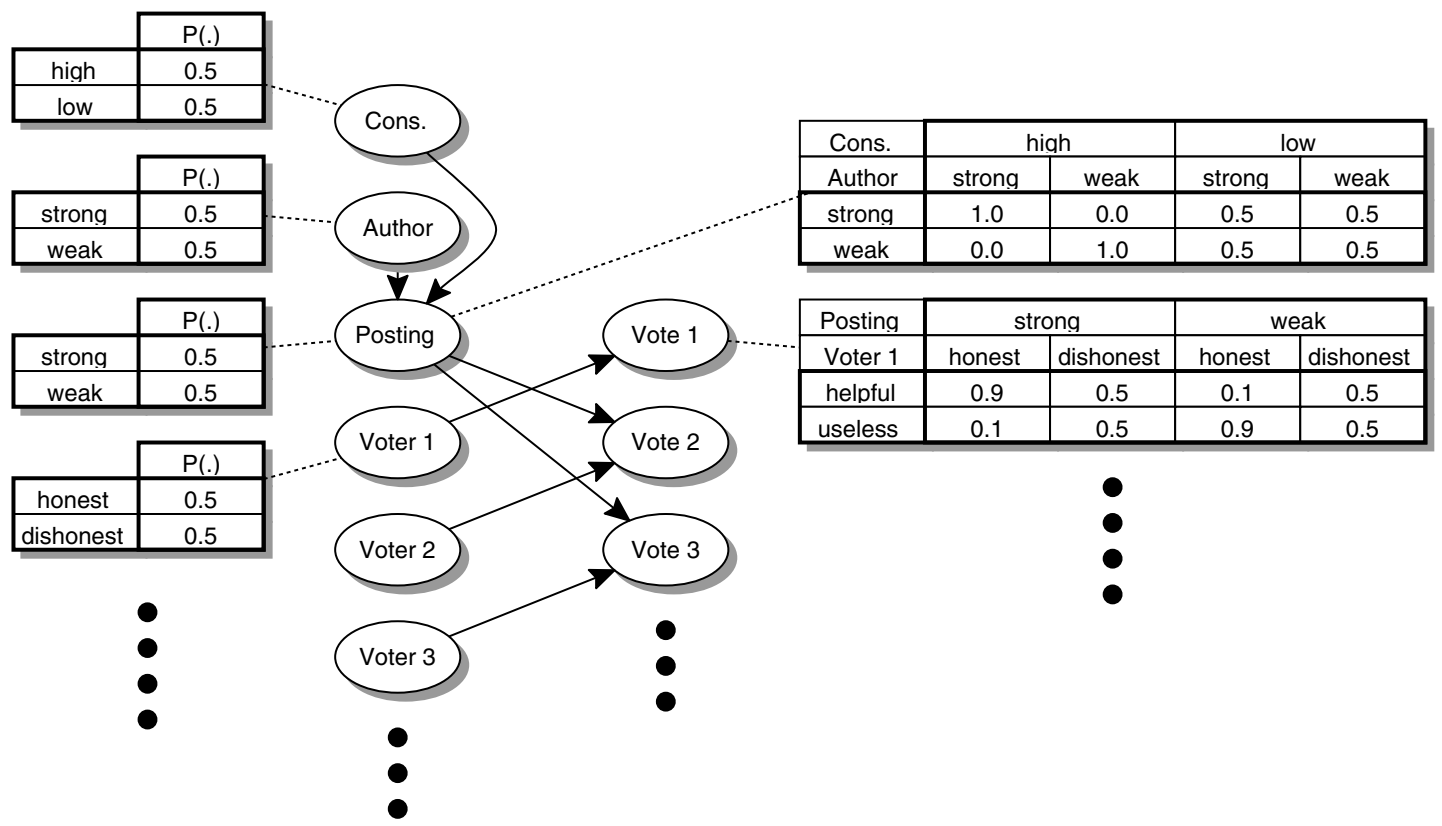

Fig. 2. Bayesian Network for Voter Honesty, Posting/ Author Quality, and Author Consistency

Two examples for Bayesian Belief Update:

- Scenario I: We start from the BBN in Fig. 2 and assume four voters altogether. Three of them rate the posting as helpful (agreeing voters), one of them rates the posting as useless (disagreeing voter). After Bayesian propagation, the following result is achieved:

- The relevance of the posting went up to $84 \%$

- The quality of the author went up to $67 \%$

- The consistency of the author remained at $50 \%$

- The honesty of the agreeing voters went up to $57 \%$ while it went down to $24 \%$ for the disagreeing voter

- Scenario II: We modify the BBN from Fig. 2 slightly: The quality of the author is now $25 \%$ initially instead of $50 \%$. The behavior of the four voters is exactly the same. In this case:

- The relevance of the posting went up to $84 \%$

- The quality of the author went up to $58 \%$

- The consistency of the author went down to $25 \%$

- The honesty of the agreeing voters went up to $57 \%$ while it went down to $24 \%$ for the disagreeing voter

\section{The Website}

\section{A. Problem Threads}

The bread and butter of the application is in allowing users to post images and descriptions of urban problems. The guidelines will state that the problems should not be of a personal nature (such as a poorly parked vehicle) and that the images should not contain any personal information (such as the licence plate of such a vehicle). All submissions will be moderated prior to posting according to these guidelines.

Fig. 3 shows the interface for the creation of a problem thread both for General Users and Power Users. Details on those user groups are given in Section V.
All users, regardless of log in status, should be able to view a list of problem threads and the associated content.

Although casual browsers (anonymous users) are not eligible to contribute (but will be able to see the content), a simple sign-up will be sufficient to become a contributor. In addition, power users are required to e.g. tag their input as comment or solution using a menu button whereas the feedback users are asked to provide feedback about the relevancy of problem threads and the helpfulness of comments and solutions, cf. Fig. 4.

\section{B. Other Design Issues}

1) Benefits of a Google Map Mashup: Users are familiar with their neighborhood and daily ways. Therefore, and in order to support social networking and shared experiences, we will consider the specification of a location on GoogleMaps per problem thread.

By this, we can measure the spatial extension of contributors and show overlaps and neighborhood with other contributors. People might be more motivated to use the system for

- collaboration on a grander scale in which multiple users post problems from a variety of locations in the city, eventually creating a mapping of the city using its problematic visions as landmarks, and/ or

- clustering with others around specific problems. Such interactions have the potential to lead to solutions and create a sense of belonging as users will most likely identify best with those who suffer from the same problematic element. An analysis will be conducted on the success of clustering around posted threads and trends that favor certain types of problems in lieu of others. 


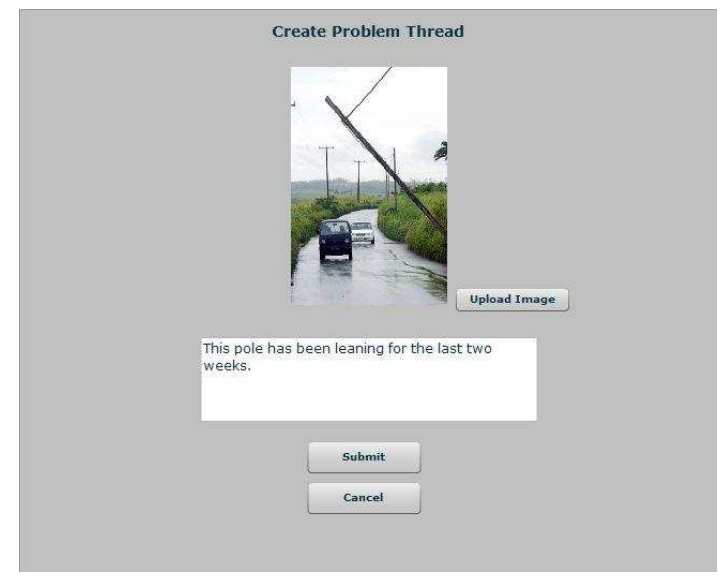

(a) Simple Interface

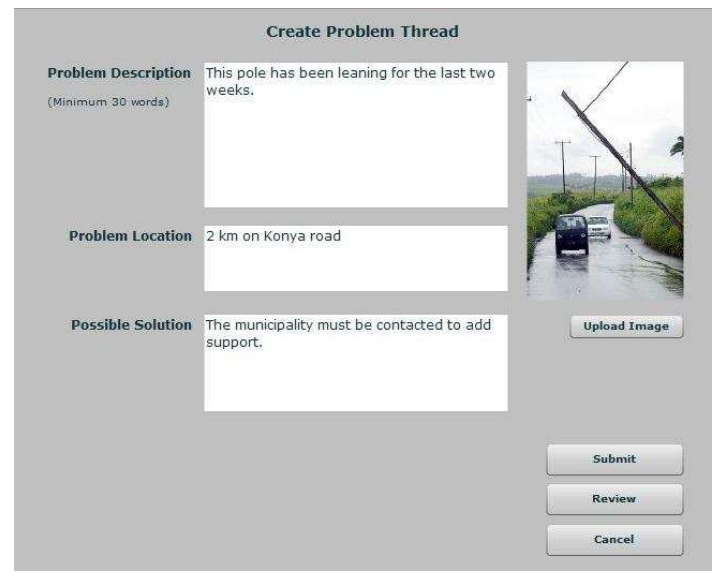

(b) Complex Interface

Fig. 3. Interface for Problem Thread Creation

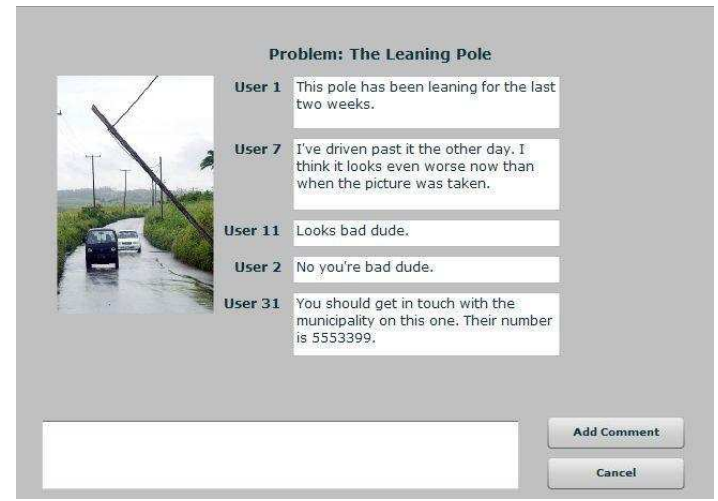

(a) Without Feedback

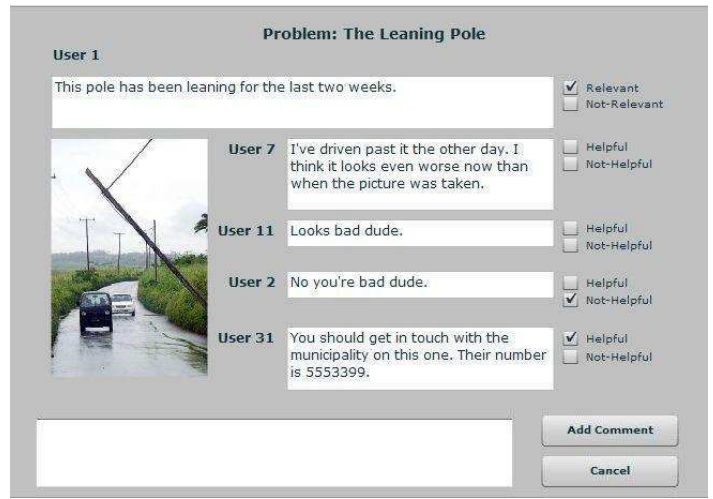

(b) With Feedback

Fig. 4. Interface for Thread Inspection, Postings and Solutions

\section{Case Study - Detailed Layout}

The two phases of the intended case study are laid out in Fig. 1. In Phase One two user groups are considered, in Phase Two those are split towards added interface functionality leaving control groups behind. Prior to Phase One - and not sketched in the figure - we will have a Phase Zero for initial community building.

\section{A. Phase Zero}

A reasonable number of users are needed in order to initiate Phase One. The recruitment phase can also be used in improving the catchiness of the web-site.

During Phase Zero, we will study the user acceptance of the web-site, the percentage of enrollment from those visiting the site and last but not least the total numbers of active users. A minimum number of users is required to kick-off the first phase of the case study.
These studies will continue throughout the entire lifetime of the web-site, but they are only used for change during Phase Zero.

A number of sample problem threads and discussions will initially be placed on the web-site in order to give visitors an idea on the mechanics of the web-site. The initial content will also serve to minimize the loss of potential members among visitors turning away in the belief that the web-site is inactive.

Among the registering users, roughly every other user will be chosen at random to give feedback on the relevance of problem threads (relevant/not relevant) and the quality of comments (helpful/not helpful). This feedback on threads and comments will be made via radio buttons but the user is not given a default state and is free not to make a selection. The results of these ratings will not be visible to the general users. This step will create two groups: General Users and Feedback Users. 


\section{B. Phase One}

During Phase I, additional users may register and Phase 0 users may drop out. New users will be assigned to either general or feedback users, based on a random selection. Although it is difficult to gauge the amount of time required for the full adoption of the web site, Phase I is expected to last between 3-8 weeks.

\section{Phase Two}

The main alteration in the second phase is the segmentation of the user-base into four groups. Roughly half of both General Users and Feedback Users will be upgraded to Power Users and Power Feedback Users respectively. Becoming a Power User will enable these members additional perks (larger volume of posts, directly contacting moderators, editing posts etc.) as well as a more complex and somewhat demanding interface for entering data. Roughly a quarter of the userbase will remain as General Users, another quarter as Feedback Users, still entering data through a simple interface. Consequently, we will have four groups in phase two: General Users, Feedback Users, Power Users and Feedback Power Users.

Having completed the user-base in the first phase, gathering of required data in the second phase should roughly take between 2-5 weeks.

\section{ANALYSIS}

For the testing of the conjectures, we need to collect data on user contributions and their quantitative development over time.

While we get quality feedback from the Feedback Users, some manual evaluation is needed as well. The validity and quality of problem threads, postings and solutions will be manually assessed by the moderators during filtering of obscenities and personal attacks. The analysis will begin with authenticating whether the posted problem truly exists at the described location. Upon conducting an on-site study, the validity of the comments and the solutions will be assigned values regarding their quality. Averaging multiple ratings may be required for a better analysis.

The amount of information on the web-site may be at a level that requires only a randomly selected number of threads ${ }^{3}$ to be analyzed.

All information (moderator entered, user entered and computed) will be stored in the database, so that the following values can be accessed as a function of time at any point of the investigation.

For the whole community:

- No. of Users (high activity/ low activity/ no activity)

- No. of Postings/ Solutions

- No. of Threads (new, active, dead)

For each user:

- User qualities, i.e. relevance, quality, consistency and honesty

\footnotetext{
${ }^{3}$ the most and least commented sets will be chosen regardless
}

- No. of Threads opened per user

- No. of Postings/ Solutions per user

For each contribution (problem thread, comment, solution, or feedback):

- Timestamp of Entry

- Timestamp of Moderator Reject/ Accept/ Evaluation

- Moderator Evaluation Result

With that, the conjectures can be investigated. F

\section{SUMMARY}

We have presented an innovative case study with a design oriented towards the automatic evaluation of the dynamics of the contributed quality. We plan to evaluate those dynamics for different users and different situations within the same website. We hope to present some results soon, but even without results, the layout of the use case is considered a meaningful contribution.

\section{REFERENCES}

[1] Jaime Arguello, Brian S. Butler, Elisabeth Joyce, Robert Kraut, Kimberly S. Ling, Carolyn Rosé, and Xiaoqing Wang. Talk to Me: Foundations for Successful Iindividual-Group Interactions in Online Communities. In CHI '06: Proceedings of the SIGCHI conference on Human Factors in computing systems, pages 959-968, New York, NY, USA, 2006. ACM Press.

[2] B. Esfandiari and S. Chandrasekharan. On How Agents Make Friends: Mechanisms for Trust Acquisition, 2001.

[3] D. Fensel, J. Hendler, H. Lieberman, and W. Wahlster, editors. Spinning the Semantic Web. MIT Press, 2003.

[4] Jim Giles. Internet Encyclopaedias go Head to Head. Nature, 438(7070):900-901, December 2005.

[5] Todd Holloway, Miran Bozicevic, and Katy Börner. Analyzing and Visualizing the Semantic Coverage of Wikipedia and Its Authors, Dec 2005.

[6] Jae W. Kim, Jiho Choi, and Kyesook Han. Relationship Support Activities, Community Commitment, and Voluntary Behaviour in Online Communities. International Journal of Internet Marketing and Advertising, 1(4):431-449, 2004.

[7] Bo Leuf and Ward Cunningham. The Wiki Way: Collaboration and Sharing on the Internet. Addison-Wesley Professional, April 2001.

[8] Robert Mccann, Anhai Doan, Vanitha Varadarajan, and Alexander Kramnik. Building data integration systems via mass collaboration, May 2003.

[9] Roland Müller. Knowledge Sharing and Trading on Electronic Market Places. PhD thesis, FU Berlin, 2005.

[10] Judea Pearl. Bayesian Networks. pages 149-153, 1998.

[11] Matthew Richardson, Rakesh Agrawal, and Pedro Domingos. Trust Management for the Semantic Web, volume 2870. January 2003.

[12] Jordi Sabater and Carles Sierra. Review on Computational Trust and Reputation Models. Artificial Intelligence Review, 24(1):33-60, September 2005.

[13] Markus Schaal. A Bayesian Approach for Small Information Trust Updates. In Proceedings of IeCCS 2006, 2006.

[14] Don Tapscott and Anthony D. Williams. Wikinomics: How Mass Collaboration Changes Everything. Portfolio Hardcover, December 2006.

[15] Fernanda B. Viegas, Martin Wattenberg, and Kushal Dave. Studying Cooperation and Conflict between Authors with History Flow Visualizations. In CHI '04: Proceedings of the 2004 conference on Human factors in computing systems, pages 575-582. ACM Press, 2004.

[16] Luis von Ahn and Laura Dabbish. Labeling images with a computer game. In CHI '04: Proceedings of the SIGCHI conference on Human factors in computing systems, pages 319-326, New York, NY, USA, 2004. ACM Press. 